\title{
Magnetic Self-healing Hydrogel from Difunctional Polymers Prepared via the Kabachnik-Fields Reaction
}

Xianzhe He ${ }^{\mathrm{a}}$, Yuan Zeng ${ }^{\mathrm{a}}$, Guoqiang Liu ${ }^{\mathrm{a}}$, Ye Tian ${ }^{\mathrm{a}}$, Yen $\mathrm{Wei}^{\mathrm{a}}$, Lingyun Zhao ${ }^{\mathrm{b}}$, Lei Yang ${ }^{\text {** }}$, Lei Tao ${ }^{\text {a* }}$

a The Key Laboratory of Bioorganic Phosphorus Chemistry \& Chemical Biology (Ministry of Education), Department of Chemistry, Tsinghua University, Beijing 100084, P. R. China.

Email: 1eitao@mail.tsinghua.edu.cn.

${ }^{b}$ Key Laboratory of Advanced Materials, Ministry of Education, Institute of Regenerative Medicine and Biomimetic Material Science and Technology, Tsinghua University, Beijing 100084, P. R. China.

${ }^{c}$ Department of Diagnostic Radiology, National Cancer Center/National Clinical Research Center for Cancer/Cancer Hospital, Chinese Academy of Medical Sciences and Peking Union Medical College, Beijing 100021, P. R. China.

Email: yangleincc@163.com.

\section{Materials}

$N$-(3-Aminopropyl) methacrylamide hydrochloride (APMA, Heowns, 98\%), poly(ethylene glycol) methyl ether methacrylate (PEGMA, Mn 950 $\mathrm{g} \mathrm{mol}^{-1}$, Sigma Aldrich Ltd.), azobisisoheptanenitrile (ABVN, J\&K, 97\%), diethylphosphite (DEPP, Aladdin, 99\%), triethylamine (TEA, Sinopharm Chemical Reagent, AR), 4fromylphenylboronic acid (4-FPBA, Soochiral Chemical Science Ltd., 98\%), bromotrimethylsilane (TMS-Br, Heowns, 95\%), $\mathrm{Fe}_{3} \mathrm{O}_{4}$ nanoparticles (Dalian Meilun Biological Technology Company), poly(vinyl alcohol) (PVA-0588, Anhui Wanwei 
Ltd.), Roswell Park Memorial Institute-1640 (RPMI-1640) culture medium (CorningCellgro), phosphate buffered saline (PBS, $\mathrm{pH} \sim 7.2-7.4,0.01 \mathrm{M}$, Solarbio), fetal bovine serum (FBS, Gibco), penicillin-streptomycin solution (Gibco), trypsin-EDTA (Gibco, 0.25\%), fluorescein diacetate (FDA, Sigma), propidium iodide (PI, 94\%, Sigma) and Cell Counting kit-8 (CCK-8, 10\%, Beyotime) were used as purchased. Methanol (Sinopharm Chemical Reagent, AR), ethanol (Sinopharm Chemical Reagent, AR) and acetonitrile (Sinopharm Chemical Reagent, AR) were purchased from commercial sources and used without further purification.

\section{Instruments}

${ }^{1} \mathrm{H}$ NMR and ${ }^{31} \mathrm{P}$ NMR spectra were obtained using a JEOL JNM-ECA400 (400 $\mathrm{MHz}$ ) spectrometer for all samples. Gel permeation chromatography (GPC) analyses of polymers were performed using water $\left(\mathrm{pH}=7.0,30{ }^{\circ} \mathrm{C}, 1 \mathrm{~mL} \mathrm{~min}^{-1}\right)$ containing 0.2 $\mathrm{M} \mathrm{NaNO}_{3}$ and $0.01 \mathrm{M} \mathrm{NaH}_{2} \mathrm{PO}_{4}$ as the eluent. The GPC system was an Agilent PLgel $5 \mu \mathrm{m}$ MIXED-C (made in GB) system containing a waters 2414 index detector. The system was calibrated with narrow molecular weight distribution polyethylene glycol standards ranging from 500 to $10^{6} \mathrm{~g} \mathrm{~mol}^{-1}$. The UV-vis spectra were measured on a Shimazu UV-2450 UV-vis spectrometer. The FT-IR spectra were recorded in a transmission mode on a Perkin-Elmer Spectrum 100 spectrometer (Waltham, MA, USA). The scanning electron microscope (SEM) photos were taken from HITACHI SU-8010. The rheology analyses were performed on a TA-AR2000ex rheometer with parallel plate geometry (diameter: $20 \mathrm{~mm}$ ). The magnetothermal effect was studied using an alternating magnetic field (AMF, Shuangping Instrument Technology), and 
the temperature was measured by an infrared thermometer (Thermo). The magnetic resonance imaging data were obtained using a Philips Achieva 3.0T TX. Cell viability was measured via CCK-8 assays by a VICTOR ${ }^{\mathrm{TM}}$ X3 PerkinElmer 2030 Multilabel Plate Reader. The histological images (muscle and liver) of mice were taken by a Nikon Eclipse $\mathrm{Ci}-\mathrm{E} / \mathrm{Ci}-\mathrm{L} / \mathrm{Ci}-\mathrm{S}$ microscope. The total bilirubin (TBIL), alanine aminotransferase (ALT), and aspartate aminotransferase (AST) levels were assessed by an automatic Biochemistry Analyzer (Sinnowa, Nanjing, China).

\section{Methods}

\subsection{Synthesis of P0 and P1}

In this study, the polymers were prepared as previous literatures. ${ }^{1}$ The precursor polymer (P0) was synthesized via a combination of the KF reaction and free radical polymerization in a one pot manner (Figure S1a). APMA (3.58 g, $20 \mathrm{mmol})$, 4-FPBA (3.29 g, $22 \mathrm{mmol})$ and TEA (3.28 g, $22 \mathrm{mmol})$ were dissolved in $30.0 \mathrm{~mL}$ of acetonitrile-ethanol (1:1, v/v). Then, DEPP (3.86 g, 28 mmol), PEGMA (9.50 g, 10 mmol) and ABVN (74.5 mg, $0.3 \mathrm{mmol}$ ) were added. The mixture was purged with nitrogen for $30 \mathrm{~min}$ followed by keeping in a $60^{\circ} \mathrm{C}$ oil bath for $12 \mathrm{~h}$. The polymerization was quenched in an ice-water bath, the mixture was dialyzed against methanol (MWCO: $3500)$ for $48 \mathrm{~h}$. The polymer P0 was obtained as a white powder after rotary evaporation (15.30 g, yield: $\sim 86 \%)$.

Then, the target polymer P1 was obtained by the hydrolysis of P0. A solution of P0 (8.00 g) in acetonitrile (200 mL) was added TMS-Br (30.00 g, $200 \mathrm{mmol})$ dropwise. The mixture was stirred at $65^{\circ} \mathrm{C}$ for $12 \mathrm{~h}$, then kept in an ice-water bath followed by 
addition of methanol $(200 \mathrm{~mL})$ dropwise and stirring for $1 \mathrm{~h}$. The volatiles were removed under vacuum and the crude was dialyzed against methanol (MWCO: 3500) for $48 \mathrm{~h}$ to get $\mathrm{P} 1$ as a white powder (6.32 $\mathrm{g}, 79 \%$ yield).

In the ${ }^{1} \mathrm{H}$ NMR spectra of P0 and P1 (Figure S1b), the distinctive peaks of the phenyl group (7.43 and $7.83 \mathrm{ppm}$ ) are clearly visible. The integral ratios between the phenyl group and the methoxyl group at the PEG chain end (3.37 ppm) are the same (P0: 3.74/3, P1: 3.75/3) and consistent with the theoretical value (4/3). Meanwhile, the methyl peaks of $\alpha$-aminophosphonate (1.14 and $1.25 \mathrm{ppm})$ in $\mathrm{P} 0$ completely disappeared in P1 and the methylene group by the amine moved from $2.49 \mathrm{ppm}(\mathrm{P} 0)$ to $2.93 \mathrm{ppm}(\mathrm{P} 1) ;{ }^{31} \mathrm{P}$ NMR spectrum significantly shifted from $25 \mathrm{ppm}(\mathrm{P} 0)$ to $9 \mathrm{ppm}$ (P1) (Figure S1b, inset). In the GPC spectra, the Mn and PDI of P1 and P0 are nearly identical, confirming its successful hydrolysis. The slightly higher Mn of P1 may be attributed to the more spreading polymer chain segments of PA structure in water. The UV-vis absorption of P1 ( 1.5) at 250-260 nm significantly enhanced compared with P0 ( 0.6). In the FTIR spectra of P1, the absorption peak at 3000-3500 nm corresponding to PA group is also enhanced compared with P0. These results suggest the successful preparation of the desired PA containing polymer after the complete hydrolysis of $\mathrm{P} 0$.

\subsection{Hydrogel preparation}

Typically, IONPs (100 mg) was suspended in a P1 solution ( $1 \mathrm{~mL}, 12 \mathrm{wt} \%$ in PBS, $\mathrm{pH} \sim 7.4)$ followed by mixing with a PVA solution ( $1 \mathrm{~mL}, 12 \mathrm{wt} \%$ in PBS, $\mathrm{pH} \sim 7.4)$. The hydrogel (Gel-1) was formed in around 10 seconds after vortex $\left(25^{\circ} \mathrm{C}\right)$. P0 was 
used to prepare a control hydrogel (Gel-0) similarly.

\subsection{Self-healing study and rheology analyses of hydrogel}

Qualitative study: Gel-1 was placed in a syringe, then pushed out through a needle (25-gauge) into a glass bottle. Photos were taken at different time points. A gelatin hydrogel $(12 \mathrm{wt} \%)$ was prepared and used as a control: Gelatin $(1.20 \mathrm{~g})$ was dissolved in a PBS solution $(\mathrm{pH} \sim 7.4,8.80 \mathrm{~mL})$, this gelatin solution was kept in a $70{ }^{\circ} \mathrm{C}$ oil bath for $6 \mathrm{~h}$, then cooled down to $25^{\circ} \mathrm{C}$ to form the desired gelatin hydrogel $(12 \mathrm{wt} \%)$.

Quantitative study: Rheology analyses were carried out to test the self-healing properties of Gel-1. A piece of Gel-1 $(\sim 1 \mathrm{~mL}, 20 \mathrm{~mm}$ diameter $)$ was put on the measuring plate of a rheometer. The moduli of Gel-1 under different strains were tested $($ frequency $=10 \mathrm{rad} / \mathrm{s})$. Then, alternate changed strains $(1 \%$ and $300 \%)$ under the same frequency $(10 \mathrm{rad} / \mathrm{s})$ were applied to Gel-1, G' and G" values were recorded.

\subsection{Magnetothermal study of P1-PVA hydrogels}

A series of P1-PVA hydrogels (2 g) with different amount of IONPs $(100,75,50$, $25 \mathrm{mg}$ ) were prepared in glass vials. These glass vials were placed in an AMF ( $\mathrm{f}=285$ $\mathrm{kHz}, \mathrm{H}=201.2 \mathrm{Oe}$ ) (Figure S4), and the temperature of this hydrogel at different time intervals were recorded by using an infrared thermometer. A P1-PVA hydrogel without IONPs was used as a blank.

\subsection{MRI study of P1-PVA hydrogels}

A series of P1-PVA hydrogels $(1 \mathrm{~mL})$ with different amount of IONPs $(0.1,0.2$, 0.3 and $0.4 \mathrm{mg}$ ) were prepared in glass vials. To simulate the testing environment in 
MRI, these vials were placed in the center of a plastic bowl filled with agar solution (5 $\mathrm{wt} \%$ in water); after the agar cooling down to form a solid, the hydrogels were fixed in the center of the agar for $T_{2}$ scanning. Both the image intensity and the quantitatively measured $\mathrm{T}_{2}$ values were recorded using a Philips Achieva 3.0T TX. A P1-PVA hydrogel without IONPs was used as a blank.

\subsection{Cell culture}

L929 cells, a fibroblast cell line from mice, were cultured at $37{ }^{\circ} \mathrm{C}$ and $5 \% \mathrm{CO}_{2}$ in a Roswell Park Memorial Institute-1640 (RPMI-1640) medium with 10\% fetal bovine serum (FBS) and 1\% penicillin and streptomycin. Culture medium was changed every day to maintain the exponential growth of the cells.

\subsection{Cytotoxicity of polymers to L929 cells}

The cytotoxicity of P1 and PVA to L929 cells was evaluated via a cell count kit-8 (CCK-8) assay. Briefly, cells $\left(\sim 1 \times 10^{5}\right.$ cells $\left./ \mathrm{mL}\right)$ were seeded in a 96 -well plate in 100 $\mu \mathrm{L}$ of culture medium. After attachment, cells were washed twice with PBS and then cultured with different concentrations of P1 and PVA for $24 \mathrm{~h}$, respectively. Cells were washed three times by PBS and then cultured in $100 \mu \mathrm{L}$ of culture medium containing $10 \% \mathrm{CCK}-8$ for $2 \mathrm{~h}$ prior to observation. The absorbance was measured under $450 \mathrm{~nm}$ by a microplate reader (VICTOR ${ }^{\mathrm{TM}}$ X3 PerkinElmer 2030 Multilabel Plate Reader). The results were compared to a positive control (cells cultured in pure medium; 100\%) and a negative control (no cells in plate; $0 \%$ ) to calculate the cell viability.

\subsection{Cytotoxicity of P1-PVA hydrogel to L929 cells via 3D cell culture}

Typically, $3 \times 10^{6}$ cells were suspended with a culture medium containing P1 (12 $\mathrm{wt} \%, 500 \mu \mathrm{L})$. Then, the cell suspension was mixed with a culture medium containing PVA $(12 \mathrm{wt} \%, 500 \mu \mathrm{L})$ to form a cell-embedded P1-PVA hydrogel. The cell-laden 
hydrogel was incubated at $37^{\circ} \mathrm{C}$ and $5 \% \mathrm{CO}_{2}$ for $24 \mathrm{~h}$ before observation. The FDA/PI double staining was used to evaluate the cell viability and the proliferation conditions (488 $\mathrm{nm}$ and $543 \mathrm{~nm}$, respectively). Z-stacks were recorded throughout the depth of the gels to verify the even distribution of the cells.

\subsection{Animal experiments}

The bio-safety of the P1-PVA hydrogel containing $50 \mathrm{mg}$ of IONPs was tested using living mice as the model animal (six BALB/C mice /group, 6 weeks old, 18-21 $\mathrm{g}$, female). All animal procedures in this study were performed in accordance with the Guidelines for Care and Use of Laboratory Animals of Tsinghua University and approved by the Animal Ethics Committee of Tsinghua University. All experimental animal procedures were performed under anesthesia, and all efforts were made to minimize suffering.

Briefly, hair on mice's waists was removed by a depilatory paste. P1 (500 $\mu \mathrm{L}, 12$ wt $\%)$ containing IONPs (50 mg) and PVA (500 $\mu \mathrm{L}, 12 \mathrm{wt} \%)$ solutions were mixed in a $2 \mathrm{~mL}$ syringe to prepare a hydrogel. This hydrogel was injected under the skin of $\mathrm{BALB} / \mathrm{C}$ mice via a needle (22-gauge). Healthy mice were used as blank. The inflammation and oedema of the skin were observed and recorded by photograph. After 3 days, mice were euthanized by carbon dioxide asphyxia. Liver and muscle tissues around hydrogel were separated and fixed in a buffered formaldehyde solution $(10 \%$, $\mathrm{w} / \mathrm{v})$, then embedded in paraffin and stained with hematoxylin and eosin (H\&E). Blood was collected and serum was separated by centrifugation $\left(3000 \mathrm{rpm}, 30 \mathrm{~min}, 20^{\circ} \mathrm{C}\right)$. The TBIL, ALT and AST values were assessed by an automatic Biochemistry Analyzer 
(Sinnowa, Nanjing, China) at $37^{\circ} \mathrm{C}$. The degradation property of the hydrogel was investigated by photographing and weighting Gel-1 under the skin at 3 days and 7 days.

\section{Supporting Data}

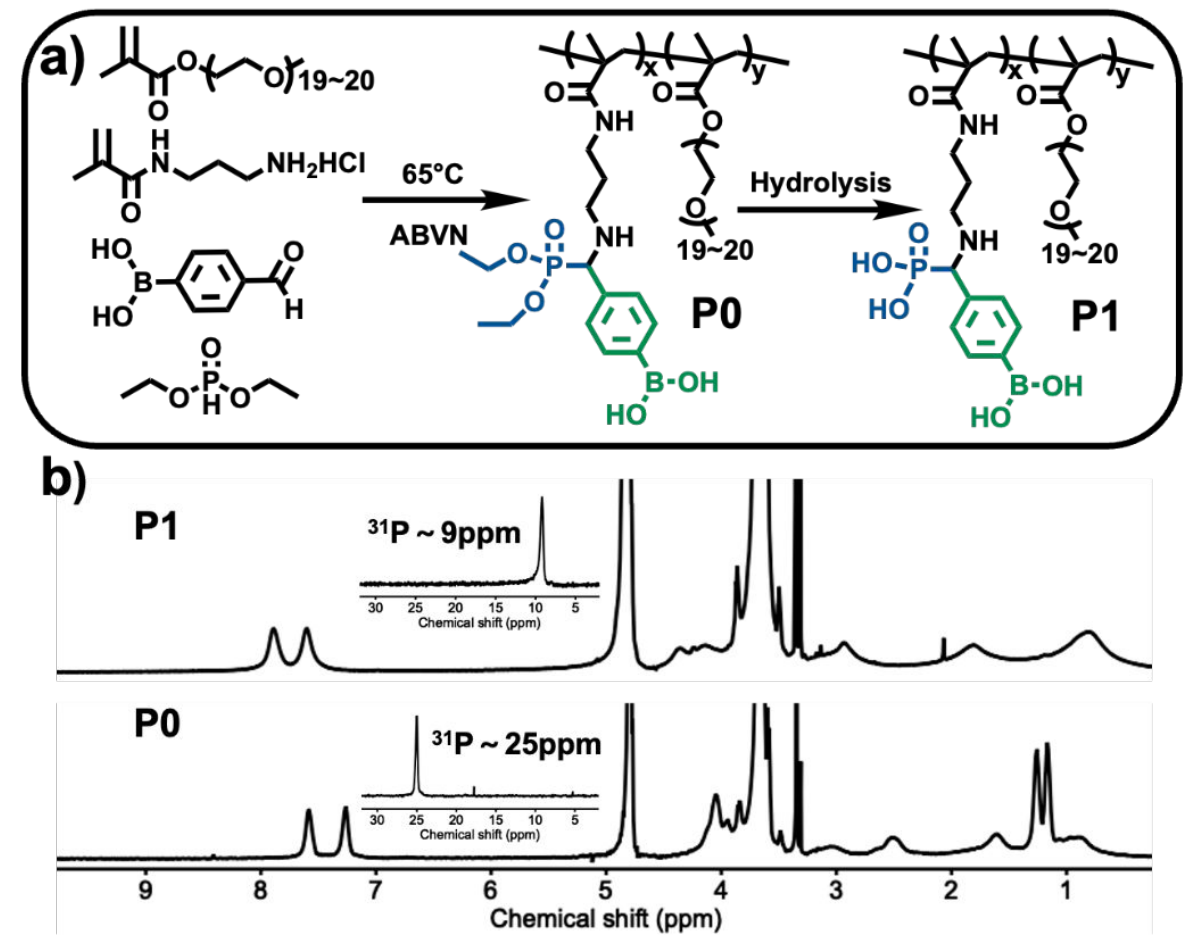

Figure S1. a) Preparation of P0 via a one-pot KF reaction/ free radical polymerization and P1 via the hydrolysis of P0; b) ${ }^{1} \mathrm{H}$ NMR spectra and ${ }^{31} \mathrm{P}$ NMR spectra (inset) of P0 and $\mathrm{P} 1$. 
a)
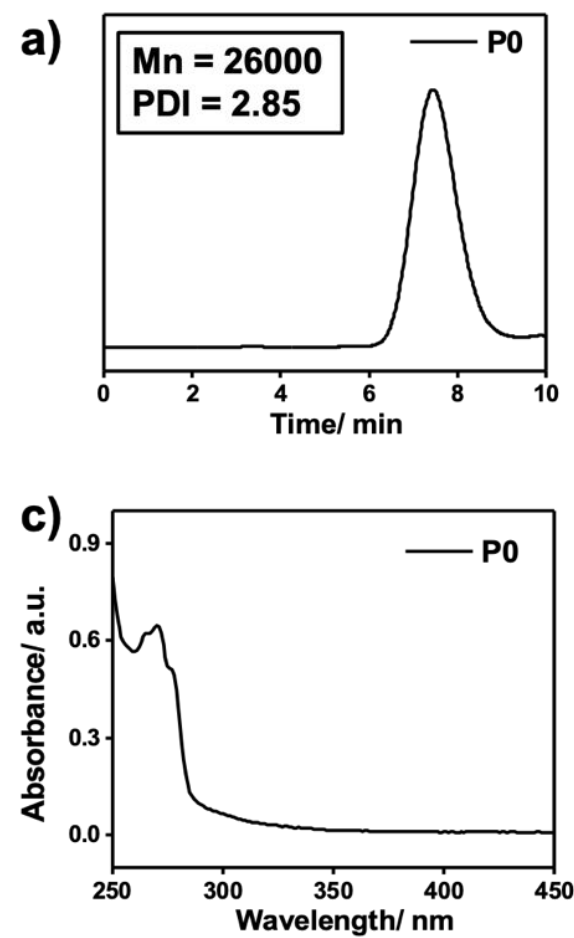

e)

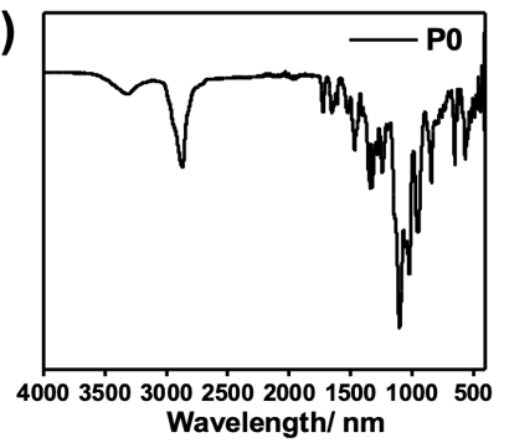

b)
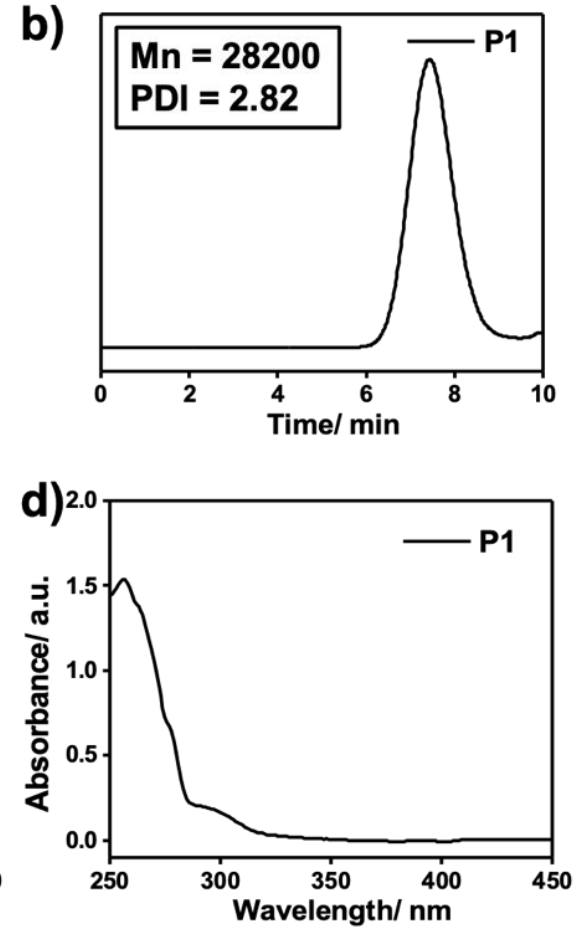

f)

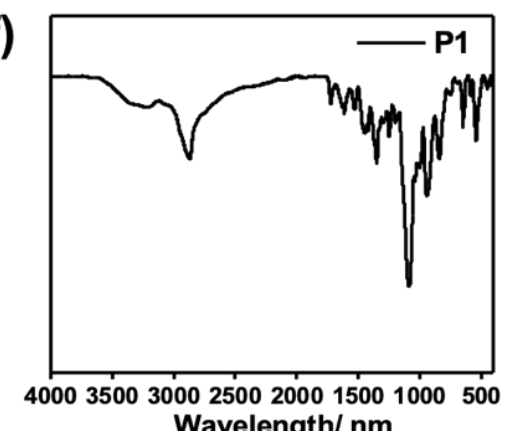

Figure S2. a, b) GPC spectra of P0 (a) and P1(b); c, d) UV-vis spectra of P0 (c) and P1 (d); e, f) FTIR spectra of P0 (e) and P1 (f).

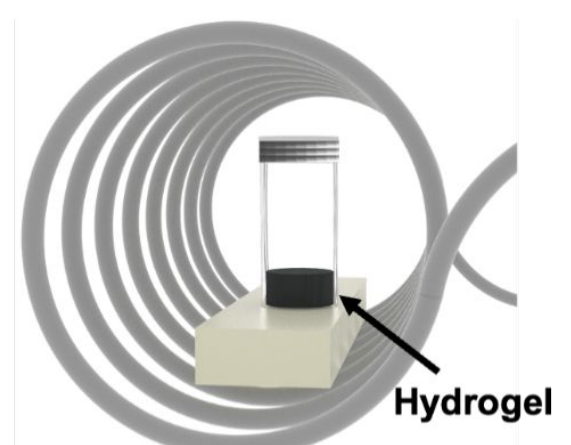

Figure S3. Illustration of the magnetothermal effect of P1-PVA hydrogels in an alternating magnetic field. 

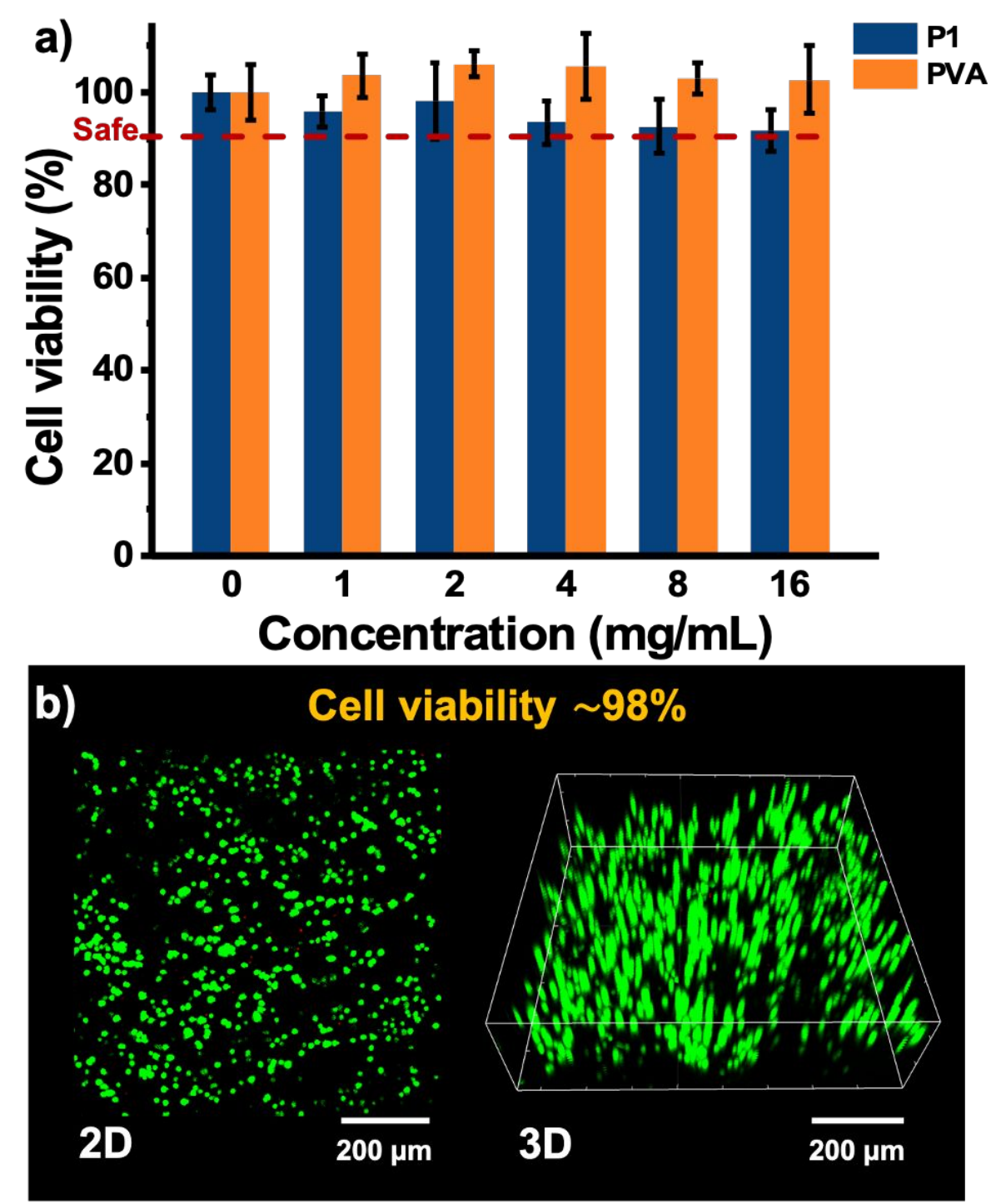

Figure S4. a) Cytotoxicity of P1 and PVA using a CCK-8 assay with a L929 cell line. Data are presented as mean $\pm \mathrm{SD}(\mathrm{n}=5)$ compared with the blank (cells in culture medium only); b) cell viability after a $24 \mathrm{~h}$ culture in a P1-PVA hydrogel via an FDA/PI assay. 

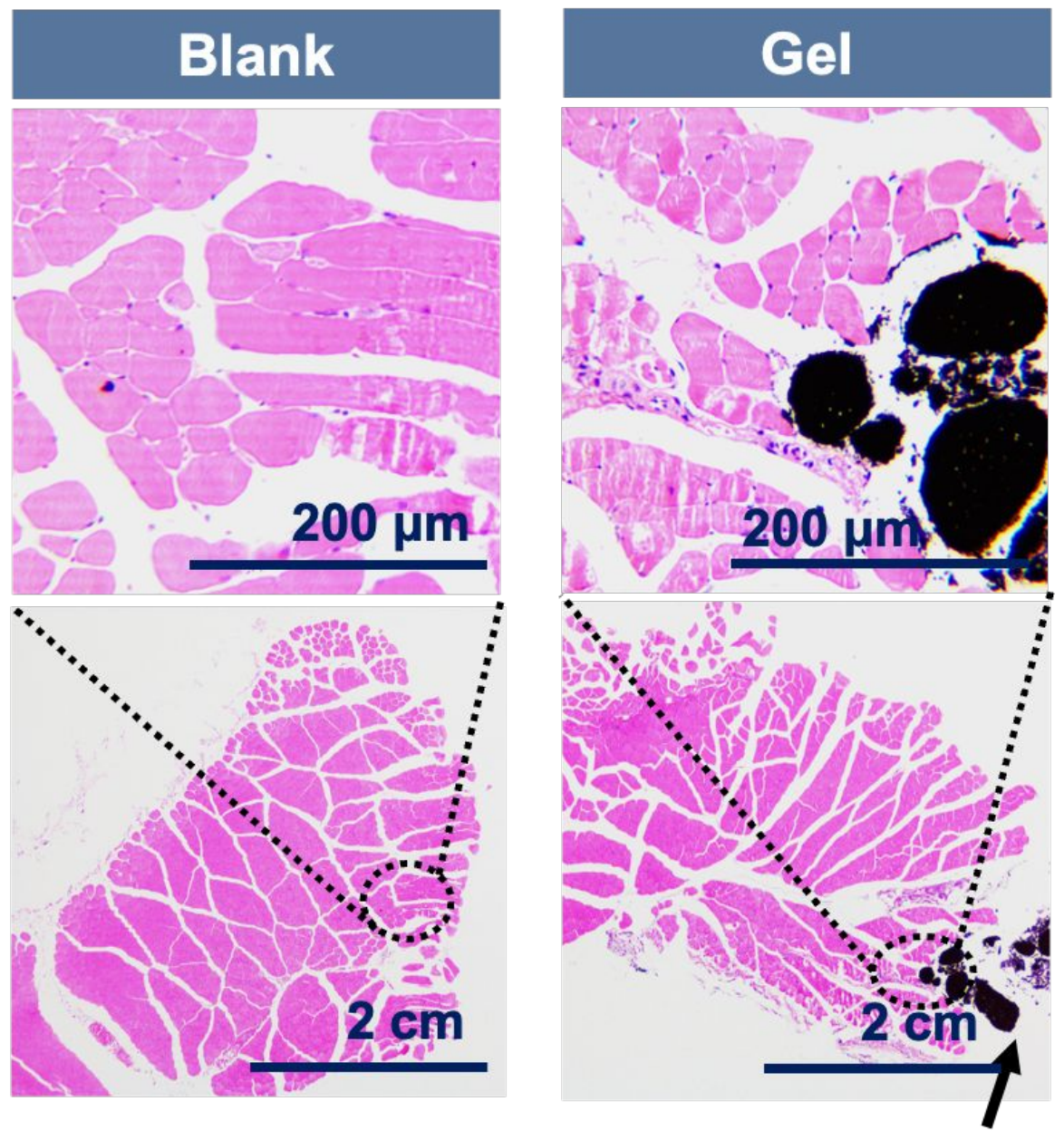

\section{Hydrogel}

Figure S5. Histological images (H\&E staining, $200 \times$ and $40 \times)$ of the tissues around the hydrogel $(\mathrm{Gel})$, muscle tissues taken from healthy mice served as a blank. 

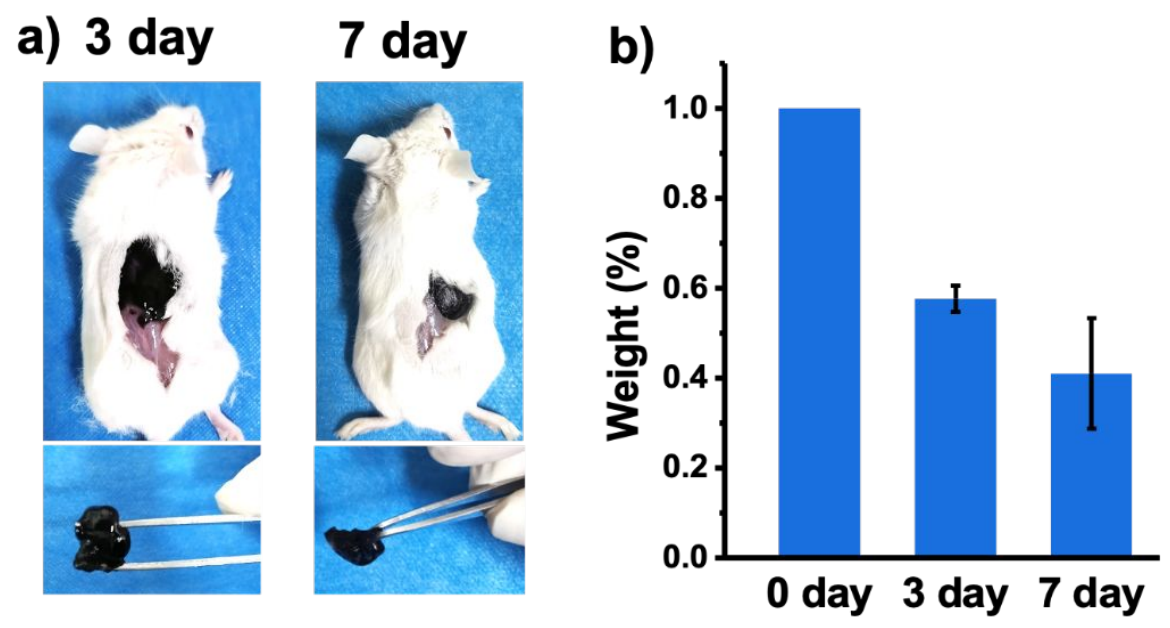

Figure S6. Degradation of the Gel-1 hydrogel under the sink of mice. a) Photographs of Gel-1 at 3-day and 7-day; b) remained Gel-1 under skin versus time, data are represented as mean $\pm \mathrm{SD}, \mathrm{n}=3$.

\section{References}

1. Tian, Y.; Zeng, Y.; Li, Y.; He, X.; Wu, H.; Wei, Y.; Wu, Y.; Wang, X.; Tao, L., Polyanionic self-healing hydrogels for the controlled release of cisplatin. Eur. Polym. J. 2020, 133, 109773. 